\title{
Host Cell Ploidy Underlying the Fungal Feeding Site Is a Determinant of Powdery Mildew Growth and Reproduction
}

\author{
Divya Chandran, Joshua Rickert, Candice Cherk, Bradley R. Dotson, and Mary C. Wildermuth \\ Department of Plant and Microbial Biology, 111 Koshland Hall, University of California, Berkeley 94720-3102, U.S.A.
}

Submitted 28 October 2012. Accepted 23 December 2012.

\begin{abstract}
Golovinomyces orontii is an obligate biotrophic powdery mildew (PM) that colonizes Arabidopsis thaliana and agronomic species. It establishes a specialized feeding structure in epidermal cells to fuel its extensive surface hyphal growth and reproduction. Previously, endoreduplication was identified in Arabidopsis mesophyll cells underlying the fungal feeding site, presumably to meet the metabolic demands imposed by the fungus. Furthermore, the cell cycle transcription factor MYB3R4 was shown to regulate this process. Herein, PM-induced endoreduplication is further characterized and three additional factors influencing host ploidy in cells underlying the fungal feeding site are identified. While mutations in $P U X 2$ and $P M R 6$ reduce basal ploidy, mutations in PMR5 (and MYB3R4) abrogate the PM-induced ploidy increase. Moreover, analysis of pmr5 microarray data suggests that PMR5 acts upstream of a MYB3R transcription factor such as MYB3R4 to control PM-induced ploidy. Induced endoreduplication occurs exclusively in mesophyll cells underlying the fungal feeding site at 5 days postinoculation, concomitant with PM reproduction. Gene copy number increases and chromatin remains decondensed, suggesting active, elevated gene expression. Cell ploidy underlying the fungal feeding site is highly correlated with the extent of PM growth and reproduction for these mutants, indicating that (induced) mesophyll cell ploidy is a PM susceptibility determinant.
\end{abstract}

Powdery mildew (PM) fungi are significant agronomic pathogens that cause disease on a wide variety of monocotyledonous and dicotyledonous plants worldwide, including cereals, tomato, grapevine, and roses (Dik et al. 2002). Susceptibility of the resource-rich model dicotyledonous plant Arabidopsis thaliana to PM fungi such as Golovinomyces orontii has greatly facilitated our understanding of host factors mediating PM colonization, growth, and reproduction (Micali et al. 2008). In addition, the use of nonhost PM fungi such as the barley-adapted Blumeria graminis f. sp. hordei on Arabidopsis has elucidated controls over nonhost PM resistance that restricts PM penetration. Similar to other PM fungi, $G$. orontii exclusively infects epidermal plant cells with defined, microscopically visible

Corresponding author: M. C. Wildermuth; Telephone: +1.510 .643 .4861 ; E-mail: mwildermuth@berkeley.edu

* The $\boldsymbol{e}$-Xtra logo stands for "electronic extra" and indicates that six supplementary tables, two supplementary figures and a supplementary data set are published online.

(C) 2013 The American Phytopathological Society stages of infection. Colonization requires germination of the conidium at approximately $2 \mathrm{~h}$ postinoculation (hpi), penetration of the epidermal cell at approximately $6 \mathrm{hpi}$, and development of the haustorial complex (the feeding structure through which the fungus acquires all its nutrients) at 1 day postinoculation (dpi) (Micali et al. 2008). The extensive surface hyphal growth and formation of asexual reproductive structures known as conidiophores occurs later, at 3+ dpi. Wind-borne conidia from the conidiophores then propagate the infection.

Forward and reverse genetic approaches have been employed to identify genes and processes mediating the PM-Arabidopsis interaction (Micali et al. 2008). Most of these genes are involved in host defense, with the affected processes referred to as resistance determinants. PM resistance determinants include genes that result in the activation of defense hormone pathways controlled by salicylic acid (SA), ethylene (ET), or jasmonic acid (JA). For example, mutations in PAD4, EDS5, ICS1, and NPRI result in reduced SA signaling and response and enhanced PM growth and reproduction (Reuber et al. 1998; Wildermuth et al. 2001) whereas constitutive or hyperresponsive activation of SA limits PM growth and reproduction in mutants such as pmr4 (Nishimura et al. 2003). Though ET and JA signaling pathways are not typically activated in response to compatible $\mathrm{PM}$ fungi, constitutive activation, as in the cev1 mutant (Ellis and Turner 2001) and AtERF1 overexpressor (Gu et al. 2002), provides PM resistance. In some mutants with enhanced resistance to PM, constitutive or PMinduced cell death is evident, with PM resistance and cell death dependent on SA (e.g., edrl-edr4, and pmr4) (Micali et al. 2008). Whatever the mechanism, cell death limits infection by PM fungi, which are obligate biotrophs. Thus, cell death is also considered a resistance determinant.

Given the intimate nature of the PM-host interaction, there are likely host compatibility factors and associated susceptibility determinants required for the growth and reproduction of the obligate biotrophic PM. This expectation is supported by the comparatively low gene number in PM fungi versus autotrophic ascomycetes and the associated loss of particular metabolic pathways, the limited host range of PM species, and correspondingly small overlap in predicted effectors for the three sequenced PM fungi (Spanu et al. 2010).

$M L O 2$, together with closely related paralogs $M L O 6$ and $M L O 12$, is a compatibility factor required for penetration of the Arabidopsis epidermal cell by G. orontii and G. cichoracearum (Consonni et al. 2006). Mildew-resistance locus o proteins (MLO) are transmembrane proteins that appear to function by suppressing early host defense responses, including rapid host actin cytoskeletal reorganization and vesicular trafficking (Eichmann and Huckelhoven 2008; Micali et al. 2008). 
Although mlo-mediated resistance is independent of SA, ET, and JA, it is not specific to PM fungi (Consonni et al. 2006).

In contrast to $M L O$ genes, which modulate PM penetration of the host epidermal cell, other genes have been identified as possible compatibility factors that affect later stages of the interaction. For example, mutants in PMR5 and PMR6 exhibit reduced hyphal growth and reproduction of the fungus, with resistance independent of known resistance determinants (above). In addition, pmr5- and pmr6-mediated resistance is specific to PM fungi, not other pathogens (Vogel et al. 2002, 2004), supporting the possibility of compatibility factors that mediate later stages of the PM-host interaction. Similar to PMR5 and PMR6, MYB3R4 and PUX2 functions affect later stage PM growth and reproduction (Chandran et al. 2009, 2010). The PM resistance of myb3r4 was attributed to a defect in PM-induced endoreduplication in mesophyll cells underlying the infected haustorial complex-containing epidermal cell (Chandran et al. 2010). Endoreduplication results from an altered cell cycle in which rounds of DNA replication occur in the absence of mitosis and cell division. It results in increased gene copy number and has been associated with enhanced metabolic capacity. Therefore, Chandran and associates (2010) proposed that induced host endoreduplication adjacent to the fungal feeding site could be used to meet the metabolic demands imposed by the obligate biotrophic PM.

Herein, we further characterize the host endoreduplication response induced by $G$. orontii and examine published PMresistant mutants with undefined modes of action to determine whether they affect the endoreduplication response. In so doing, we identify three factors-PUX2, PMR5, and PMR6-in addition to MYB3R4 that contribute to induced mesophyll cell ploidy at the PM feeding site. These three factors were not previously known to affect cell ploidy but, similar to MYB3R4, are anticipated to affect cell cycle progression from $\mathrm{G} 2$ to mitosis and cell division. Further analysis of PMR5 indicates that it operates in the same transcriptional network as PMR6 to integrate differentiation and cell cycle responses, the misregulation of which affects (induced) host mesophyll cell ploidy and PM susceptibility. Because induced mesophyll cell ploidy is highly correlated with the extent of PM growth and reproduction for the wild type (WT) and mutants in MYB3R4, $P U X 2, P M R 5$, and PMR6, it acts as a PM susceptibility determinant.

\section{RESULTS}

Host endoreduplication is induced specifically in mesophyll cells underlying the fungal feeding site at $5 \mathrm{dpi}$, the external growth and reproduction phase of the PM.

As leaves develop and expand, individual cells in a leaf exhibit a range of ploidy (Melaragno et al. 1993). Ploidy distribution plots allow assessment of changes in cell ploidy and the ploidy index provides a single value that integrates the ploidy distribution information. Ploidy index $=(\% 4 \mathrm{C}$ nuclei $\times 1)+$ $(\% 8 \mathrm{C}$ nuclei $\times 2)+(\% 16 \mathrm{C}$ nuclei $\times 3)+(\% 32 \mathrm{C}$ nuclei $\times 4)+$ $(\% 64 \mathrm{C}$ nuclei $\times 5)$. To systematically determine the phase of the G. orontii-Arabidopsis interaction associated with PM-induced endoreduplication, we measured PM-induced DNA ploidy distributions and calculated ploidy indices for mature,
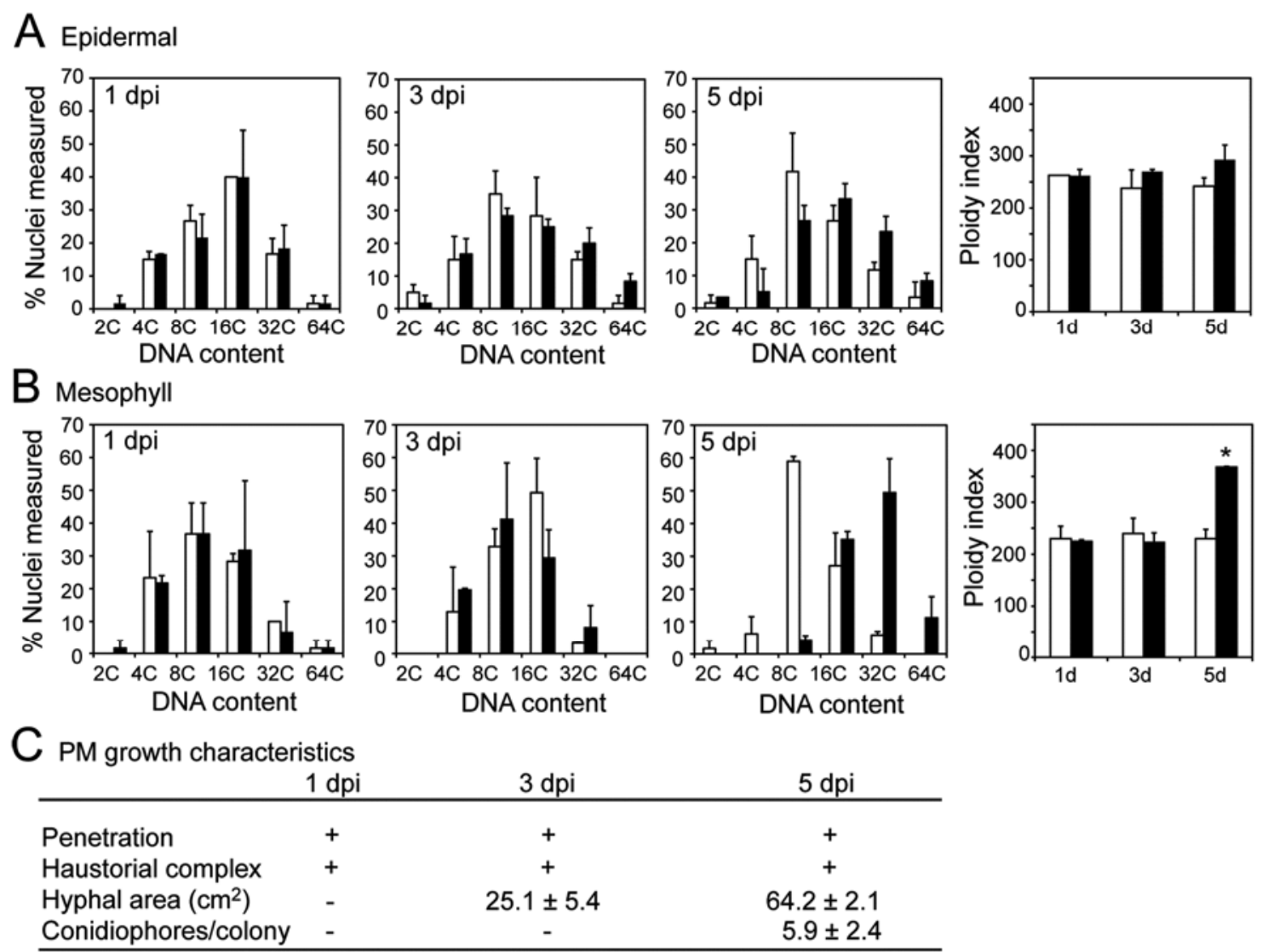

$1 \mathrm{dpi}$

\section{$5 \mathrm{dpi}$}

\begin{tabular}{ccc}
+ & + & + \\
+ & + & + \\
- & $25.1 \pm 5.4$ & $64.2 \pm 2.1$ \\
- & - & $5.9 \pm 2.4$ \\
\hline
\end{tabular}

Fig. 1. Powdery mildew (PM)-induced ploidy is specific to underlying mesophyll cells at 5 days postinoculation (dpi), the external growth and reproduction phase. Ploidy distribution of A, epidermal and B, mesophyll cells at the site of PM infection at 1, 3, and 5 dpi. Uninoculated (white bar), Inoculated (black bar). Two independent experiments gave similar results. $N \geq 30$ nuclei per experiment; shown is average ( \pm standard deviation [SD]); $*$ indicates $P$ value $<0.05$ using unpaired Student $t$ test. C, Associated PM growth characteristics; \pm SD; $+=$ present and $-=$ absent; hyphal area and conidiophores per colony were quantified from 15 isolated fungal colonies. 
fully expanded leaves of $A$. thaliana WT ecotype Columbia-0 (Col-O) at 1, 3, and $5 \mathrm{dpi}$, corresponding to i) haustorial complex development, ii) external hyphal growth and branching, and iii) sustained external hyphal growth and conidiophore production, respectively (Fig. 1). To investigate the cell-type specificity of the response to inoculation, we performed ploidy measurements for epidermal cells at the site of infection, consisting of the haustorial complex-containing epidermal cell and two neighboring epidermal cells, and for three mesophyll cells underlying the haustorial complex-containing epidermal cell. Ploidy measurements made on parallel epidermal and mesophyll cells from leaves of uninoculated plants served as controls.

As anticipated, the ploidy distribution and ploidy index for cells from control fully expanded mature leaves of plants grown under consistent, nonstressed (uninoculated) conditions did not change over the 5-day time frame of our experiments (Fig. 1). In response to PM, ploidy did not change significantly in epidermal cells at 1, 3, or 5 dpi (Fig. 1A). By contrast, in mesophyll cells underlying the fungal feeding structure, there was a clear increase in the number of $16 \mathrm{C}, 32 \mathrm{C}$, and $64 \mathrm{C}$ mesophyll cells at 5 dpi from a mean of $8 \mathrm{C}$ in mesophyll cells of control leaves (Fig. 1B). On average, 96\% ( \pm 1.4$)$ of underlying mesophyll cells had $\geq 16 \mathrm{C}$ at 5 dpi with PM compared with $33 \%$ ( \pm 8.5$)$ in controls (Supplementary Table S1). Furthermore, 61\% ( \pm 3.8$)$ had $\geq 32 \mathrm{C}$ nuclei at 5 dpi compared with only $6 \%( \pm 1.0)$ in uninoculated leaves. Consistent with the ploidy distribution, the mesophyll ploidy index exhibited a statistically significant increase only at 5 dpi (Fig. 1B). No significant alteration in mesophyll cell ploidy was observed at 1 or 3 dpi (Fig. 1B).

We did not observe a statistically significant increase in ploidy index for epidermal cells at 5 dpi (Fig. 1A). In a previous study with Arabidopsis, nuclei of haustorial complex-containing epidermal cells at approximately $1 \mathrm{dpi}$ with $G$. cichoracearum were observed to be roughly twice as large as that of uninfected cells (Koh et al. 2005). However, this observation did not take into account the considerable natural variation in Arabidopsis leaf epidermal cell ploidy and size (Melaragno et al. 1993). To specifically investigate whether the haustorial complex-containing epidermal cell exhibited a significant increase in ploidy, we separately examined the haustorial complex-containing epidermal cell and the two neighboring cells at
20 infection sites. Neither nuclear volume, cell size, nor mean $\mathrm{C}$ values differed significantly and reproducibly for the haustorial-complex-containing compared with neighboring epidermal cells (Supplementary Table S2). By contrast, underlying mesophyll nuclear and cell volumes increased, on average, 2.6-fold and 1.4-fold, respectively, at 5 dpi compared with uninoculated controls, consistent with the increase in mean $\mathrm{C}$ value from 1.9 (8C) to $3.2(32 \mathrm{C})$.

Taken together, our results show that induced endoreduplication is not associated with earlier phases of fungal development, including penetration, primary haustorial complex development or accommodation, or initial surface hyphal growth and branching, but with later-stage extensive PM external hyphal growth and reproduction (Fig. 1). Furthermore, DNA content quantified by 4',6-diamidino-2-phenylindole (DAPI) staining is proportional to nuclear size and chromatin remains largely decondensed, with a relative heterochromatin fraction (RHF) of $7 \%$ in both endoreduplicated and control cells (Supplementary Fig. S1B), suggestive of active gene expression.

\section{Host mesophyll ploidy \\ is altered in four PM-resistant mutants.}

We previously showed that myb3r4 mutants lacking the PMinduced endoreduplication response support less fungal growth and reproduction at 5 dpi (Chandran et al. 2010). Therefore, we hypothesized that other Arabidopsis mutants exhibiting PM resistance phenotypes may also be compromised in the endoreduplication response. To test this possibility, we focused on a set of mutants for which resistance is i) associated with reduced hyphal growth and reproduction, not fungal penetration or haustorial complex development; ii) independent of cell death; and iii) when assessed, independent of SA, ET, or JA signaling pathways. PM resistance phenotypes of these loci were previously confirmed by either complementing the mutation with the WT gene (Vogel et al. 2002, 2004) or using two independent mutants per locus (Chandran et al. 2009, 2010).

DNA ploidy levels in underlying mesophyll cells at 5 dpi along with control mesophyll cells from leaves of uninoculated plants were measured for WT Col-O, myb3r4 (Chandran et al. 2010), pux2 (Chandran et al. 2009), pmr6 (Vogel et al. 2002), and pmr5 (Vogel et al. 2004) mutant plants. WT plants exhib-
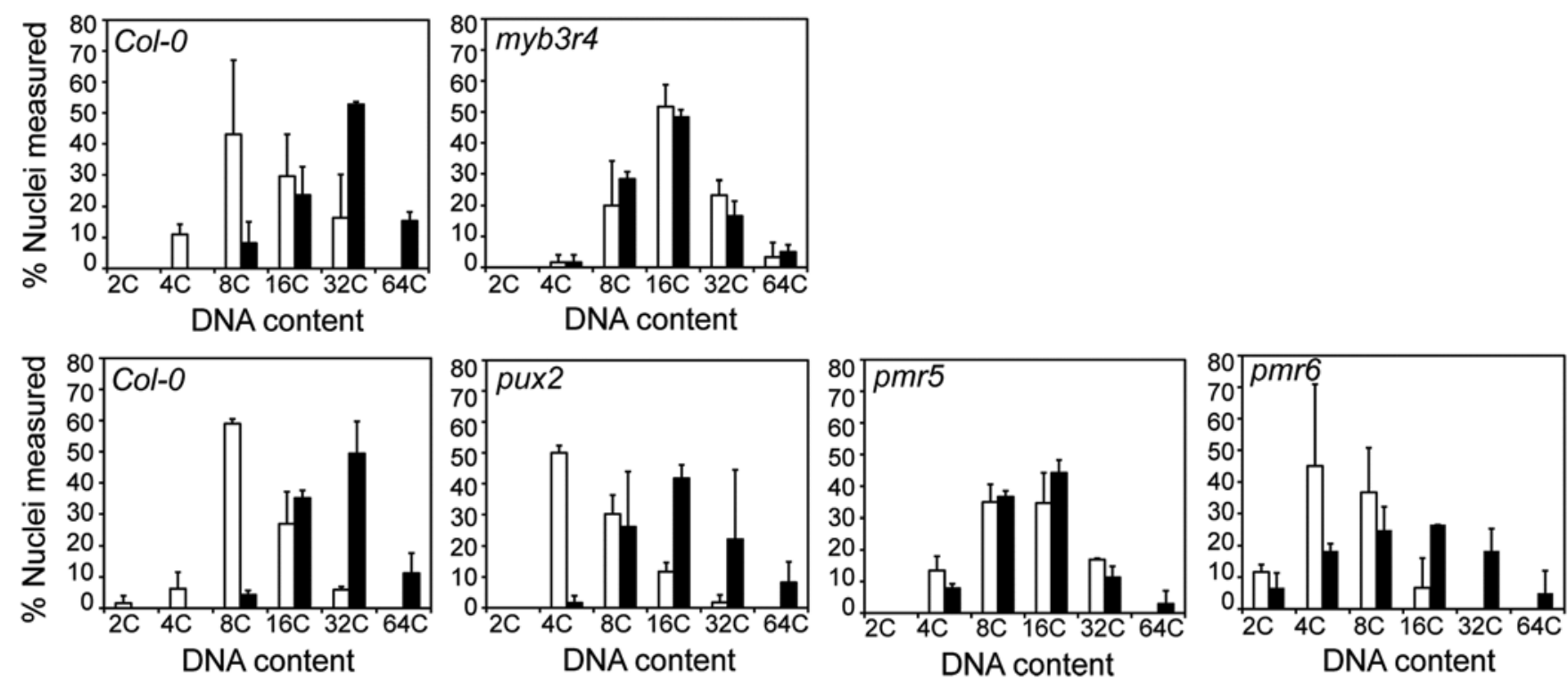

Fig. 2. Ploidy distribution of mesophyll cells at the powdery mildew infection site is altered in four mutants compared with the wild type at 5 days postinoculation. Uninoculated (white bar); Inoculated (black bar). Two independent experiments gave similar results. $N \geq 30$ nuclei per experiment; shown is average ( \pm standard deviation). 
ited a statistically significant PM-induced shift in mesophyll cell ploidy (Figs. 2 and 3A). By contrast, myb3r4 and pmr5 did not exhibit an increase in mesophyll cell ploidy distribution or ploidy index at 5 dpi with PM (Figs. 2 and 3A). To look at the extent of PM-induced change in ploidy at $5 \mathrm{dpi}$, we calculated the infected/uninfected ploidy index ratio. The WT had an infected/uninfected ploidy index ratio of $1.6 \pm 0.2$, while $m y b 3 r 4$ and pmr5 had ploidy index ratios close to $1(1.0 \pm 0.1$ and $1.1 \pm 0.1$, respectively). Though uninfected myb3r4 and pmr5 ploidy may appear to be slightly higher than WT, there was no statistically significant $(P \leq 0.05)$ difference in the uninfected ploidy index (Fig. 3A) or the percentage of mesophyll cells having $\geq 16 \mathrm{C}$ nuclear DNA content compared with the WT (data not shown).

In contrast to myb3r4 and pmr5, pmr6 and pux2 exhibited a statistically significant PM-induced shift in cell ploidy similar to that of the WT (Figs. 2 and 3A). However, the basal (uninfected) mesophyll cell ploidy in mature leaves of pmr6 and pux2 was significantly lower than the WT (Fig. 3A). The ploidy distribution was shifted in these mutants (Fig. 2), with basal median $\mathrm{C}$ values for pmr6 and pux 2 mesophyll cells of $4 \mathrm{C}$, compared with $8 \mathrm{C}$ for WT. Post inoculation, the median $\mathrm{C}$ value increased to $32 \mathrm{C}$ for WT, with pux2 and pmr6 increasing to $16 \mathrm{C}$ and $8-16 \mathrm{C}$, respectively.

\section{Host mesophyll ploidy underlying the fungal feeding site is a determinant of PM growth and reproduction.}

The pmr6 and pux2 mutants exhibited a PM-induced increase in mesophyll cell ploidy similar to the WT (Fig. 3A);

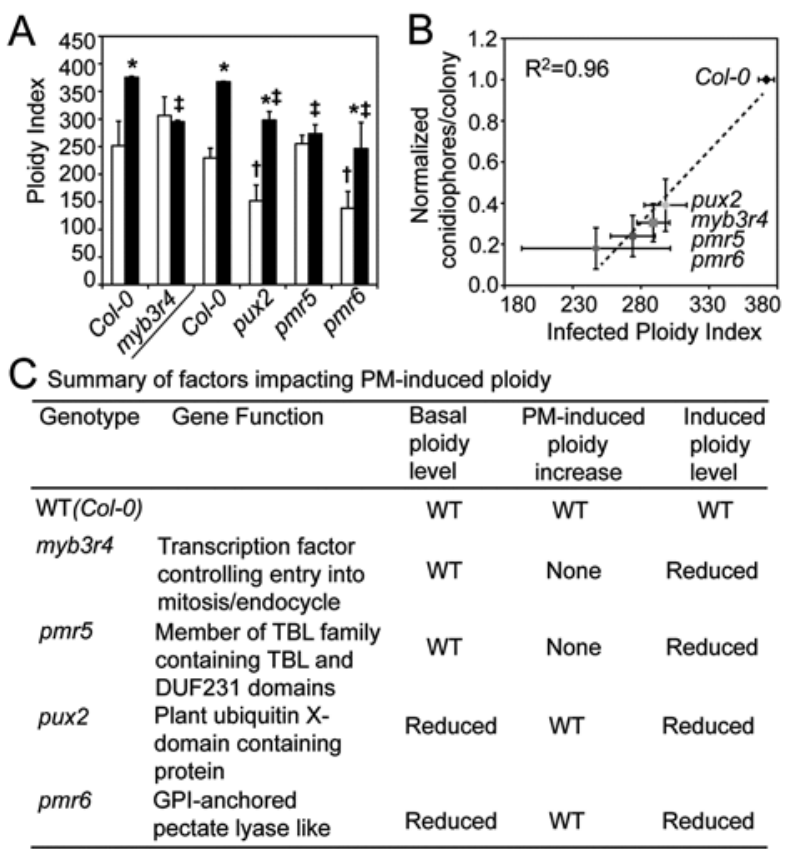

Fig. 3. Powdery mildew (PM) growth and reproduction correlates with induced mesophyll ploidy, a susceptibility determinant. A, Average ploidy index ( \pm standard deviation) at 5 days postinoculation (dpi) for mutants and parallel wild-type (WT) control. Uninoculated (white bar), Inoculated (black bar). Two independent experiments gave similar results. $N \geq 30$ nuclei per experiment; $P$ value $<0.05$, unpaired $t$ test for inoculated versus uninoculated (*) with Welch's correction applied to Col-O and pmr6 to account for unequal group variances; $P$ value $<0.05$, nonparametric analysis of variance Kruskal-Wallis test with Dunn's multiple comparison posttest for uninoculated WT versus mutant $(\dagger)$ and inoculated WT versus mutant ( $\$)$. B, Normalized conidiophores per colony at 5 dpi from two to four independent experiments compared with infected ploidy index. $R^{2}$ determined by linear regression, $y=0.007 x-1.63$. C, Summary of factors impacting PM-induced ploidy. therefore, PM resistance in pmr6 and pux2 cannot be attributed to deficient induction of localized host mesophyll cell endoreduplication. However, because the basal (uninfected) mesophyll cell ploidy of pmr6 and pux 2 was significantly lower than the WT, the final (induced) ploidy for the mesophyll cells underlying the fungal feeding site at 5 dpi was significantly lower in pmr6 and pux2 than the WT (Fig. 3A). This suggested that the final (induced) mesophyll ploidy might determine the extent of PM growth and reproduction. Therefore, we plotted normalized conidiophores per colony, a quantitative indicator of the later PM external growth and reproduction phase, versus post-inoculation mesophyll ploidy index for the WT and this mutant set. We found that the extent of PM growth and reproduction was highly correlated with host mesophyll cell ploidy underlying the fungal feeding site $\left(R^{2}=0.96\right)$ (Fig. 3B). Normalized conidiophores per colony at 5 dpi was also highly correlated with percent mesophyll cells with nuclei $\geq 16 \mathrm{C}\left(R^{2}=\right.$ 0.91) (Supplementary Fig. S2). By contrast, normalized conidiophores per colony was not correlated with either uninfected ploidy index or infected/uninfected ploidy ratio (data not shown). These findings support host mesophyll ploidy underlying the fungal feeding site as a determinant of PM external growth and reproduction and identify four genes that impact this process (Fig. 3C). Furthermore, this relationship can be described using a linear equation (Fig. 3B).

\section{Microarray analysis of pmr5 suggests that PMR5 acts as a negative regulator of induced mesophyll leaf cell ploidy via a MYB3R transcription factor.}

Using $p m r 5$, we sought to further elucidate the regulation of PM-induced endoreduplication. Upon PM infection, a clear increase in nuclear size and intensity of DAPI staining was observed in mesophyll cells underlying haustorial complexcontaining epidermal cells in WT leaves but not in pmr5 (Fig. 4A). Accordingly, there was no increase in mesophyll cell host ploidy with infection (Figs. 2 and 3A), and pmr5 supported few to no conidiophores per colony while WT averaged 5.5 conidiophores/colony (Fig. 4B), in agreement with previous results (Vogel et al. 2004).

To ascertain the mechanism by which PMR5 may regulate PM-induced endoreduplication, we analyzed Affymetrix ATH1 microarray data of pmr5 and WT Col-0 leaves. Genes with a statistically significant and $\geq 1.5$-fold change in expression in pmr5 versus the WT totaled 163, with 88 genes exhibiting enhanced expression (referred to as upregulated) and 75 genes exhibiting reduced expression (referred to as downregulated) in pmr5 compared with the WT (Supplementary Data set). Though up- and downregulated terms are used, transcript abundance reflects both transcription and mRNA degradation. Quantitative reverse-transcriptase polymerase chain reaction (qPCR) on selected genes gave results similar to that of the microarray (Supplementary Table S3). We used Biomaps in Virtual Plant (Katari et al. 2010) to identify functional processes significantly enriched in the pmr5 dataset. Genes upregulated in pmr5 belong to four major Munich Information Center for Protein Sequences functional terms: Interaction with the Environment, Tissue Differentiation, Organ Differentiation, and Cell Cycle and DNA Processing, all with $P$ values $\leq 5 \mathrm{E}$ 04 (Fig. 4C). No functional terms were statistically significant in the downregulated dataset.

Over-represented cis-acting regulatory element motifs in 1$\mathrm{kb}$ upstream promoter regions of genes with altered expression in pmr5 were ascertained using Pscan (Zambelli et al. 2009) (Supplementary Table S4). The associated transcription factor core binding sites were the Arabidopsis homeobox (ATHB) HD-ZIP element CAATNATT(G/A) and the mitosis-specific activator (MSA)-like element AACGG. Of the 163 total differ- 
A
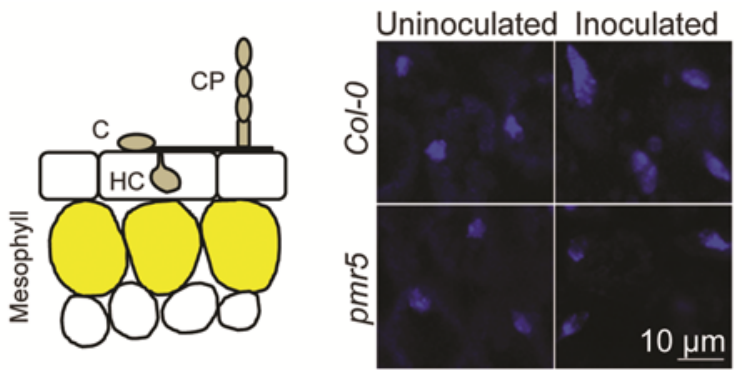

B
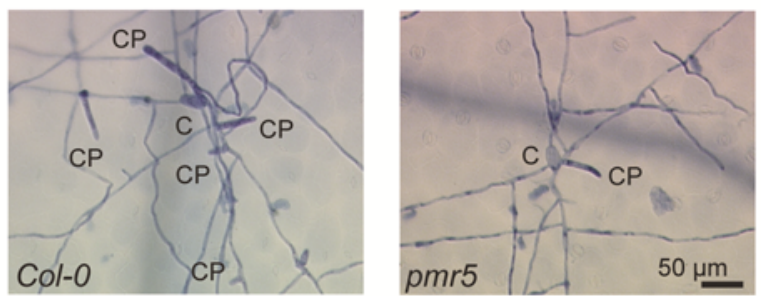

C

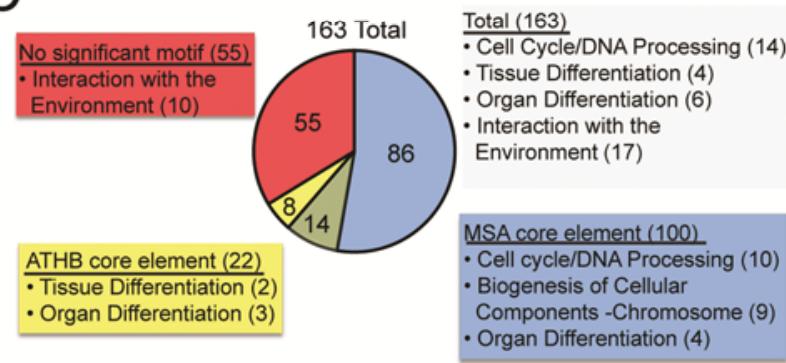

D

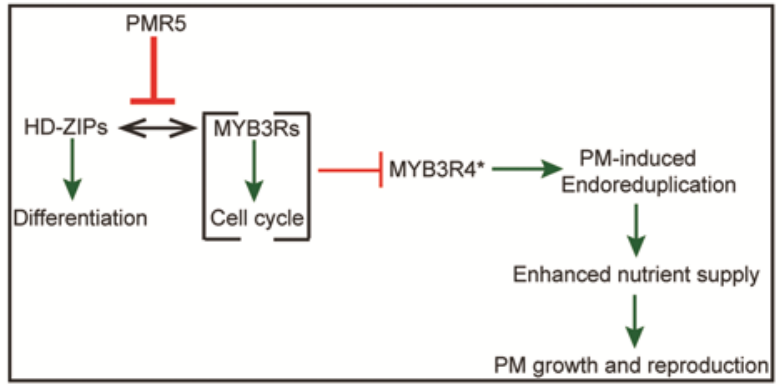

Fig. 4. PMR5 modulates cell cycle and differentiation responses to promote localized powdery mildew (PM)-induced endoreduplication and $\mathrm{PM}$ growth and reproduction in mesophyll cells underlying the haustorial complex-containing epidermal cell, A, shown in yellow with 4',6diamidino-2-phenylindole-stained nuclei of haustorial complex-underlying and control mesophyll cells. B, Conidiophores (CP) per colony with germinated conidia (C). C, Genes with statistically altered $\geq 1$.5-fold change expression in pmr5 versus wild-type grouped by shared cis-acting regulatory element, with associated statistically enriched functional categories. Green fraction indicates overlap between yellow and blue fractions on the pie chart. D, Proposed model of PMR5 function in PM susceptibility. Microarray data indicate that PMR5 acts upstream of cell cycle and differentiation responses via HD-ZIP and MYB3R transcription factors to promote PM-induced endoreduplication. Given the proposed role of un(der)-phosphorylated (*) MYB3R4 in promoting endoreduplication (Chandran et al. 2010), PMR5 likely facilitates this function of MYB3R4 at the PM-infection site, resulting in localized endoreduplication in cells underlying the fungal feeding site. This endoreduplication could, in turn, enhance the metabolic capacity of these cells, allowing for enhanced nutrient supply to the fungus and increased PM growth and reproduction. Double arrow indicates potential for bidirectional integrated crosstalk because 14 PMR5-impacted genes contain cisacting regulatory elements bound by both HD-ZIP and MYB3R transcription factors. PMR5 low-level repression of basal defense responses (e.g. by salicylic acid and ethylene) is not shown in model because previous double-mutant analyses found these pathways did not contribute to $\mathrm{pmr} 5$ resistance (Vogel et al. 2004) entially regulated genes in the pmr5 dataset, 100 contained at least one MSA element and 22 at least one ATHB core element (Fig. 4C).

Functionally, genes with the MSA core element in their promoter were statistically enriched in the categories Cell Cycle/ DNA Processing, Biogenesis of Cellular Components (chromatin structure), and Organ Differentiation (Fig. 4C). The MSA element is bound by MYB3R transcription factors, including MYB3R4 (Haga et al. 2007), of which there are five in Arabidopsis. No MYB3R genes exhibited altered expression in whole leaves of $p m r 5$ versus the WT. However, we previously reported that MYB3R4 was specifically induced at the PM infection site at $5 \mathrm{dpi}$ and that this induction could not be detected when whole leaves were analyzed (Chandran et al. 2010). Consistent with its role as a transcriptional regulator of mitosis and endoreduplication (Chandran et al. 2010; Haga et al. 2007), the top 300 ATH1 genes co-expressed with MYB3R4 identified using ATTED-II (Obayashi et al. 2009) were most highly enriched in the Cell Cycle/DNA Processing category $(P$ value $=4 \mathrm{E}-19)$.

Genes with at least one ATHB core element in their promoter were statistically enriched in the categories Tissue and Organ Differentiation (Fig. 4C). The ATHB core element is bound by HD-ZIP transcription factors. Of these, PHABULOSA/ ATHB14, PHAVULOTA/ATHB9, and REVOLUTA/IFL1 exhibited enhanced expression in $p m r 5$ compared with the WT and play known roles in tissue and organ differentiation, including leaf development (Byrne 2005). We found genes involved in Organ Differentiation, including PHABULOSA, contain both ATHB and MSA core elements in their promoters, indicative of the integration of cell cycle and differentiation responses.

The remaining genes without an MSA or ATHB core element in their promoter were enriched in the category Interaction with the Environment. These 10 genes included individuals associated with diverse hormone signaling pathways (e.g., SA, ET, gibberellin, and auxin), including the SA biosynthetic gene ICS1 and the ET biosynthetic gene ACS4. However, no particular known defense pathway was strongly altered. Similarly, the full set of 17 genes in the Interaction with the Environment category for the $p m r 5$ dataset was not strongly associated with any one hormone or defense pathway (Supplementary Table 5).

\section{DISCUSSION}

\section{Induced mesophyll host ploidy-a PM} susceptibility determinant.

In the G. orontii-Arabidopsis interaction, induced endoreduplication was not associated with earlier phases of fungal development, including penetration, primary haustorial complex development or accommodation, or initial hyphal growth and branching, but with later-stage extensive PM external hyphal growth and reproduction (Fig. 1). It occurred exclusively in mesophyll cells underlying the haustorial complex-containing

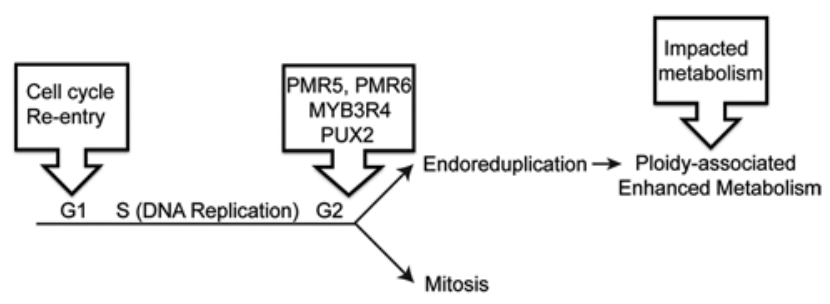

Fig. 5. Cell cycle re-entry, DNA replication, and lack of G2/M progression to mitosis and cell division mediate (infected) mesophyll ploidy levels. Powdery mildew compatibility factors described herein appear to act at the G2/M transition. 
epidermal cell, with a mean increase from $8 \mathrm{C}$ to $32 \mathrm{C}$ at $5 \mathrm{dpi}$ accompanied by a proportional increase in nuclear volume (2.6-fold) and cell expansion (1.4-fold increase in cell volume). Chromatin remained highly decondensed, with a mean RHF of $7 \%$, similar to $8 \%$ reported for Arabidopsis rosette leaves of similarly aged and grown plants (Tessadori et al. 2007) and consistent with active gene expression.

$G$. orontii-induced endoreduplication in mesophyll cells underlying the fungal feeding site occurred during the extensive surface growth and reproduction phase of PM infection (Fig. 1) and is likely to support the metabolic demands imposed by the fungus. When PM-induced mesophyll endoreduplication was abrogated, as in myb3r4 and pmr5, PM growth and reproduction was reduced (Fig. 3). In addition, when basal mesophyll ploidy was low, as it was for pux2 and pmr6, the final PM-induced ploidy (e.g., from two rounds of endoreduplication) appeared insufficient to support robust fungal growth and reproduction. Similarly, reduced host ploidy at the root nematode feeding site or in symbiotic cells resulted in reduced nematode growth or symbiosome development and nitrogen fixation, respectively (De Almeida Engler et al. 2012; Vinardell et al. 2003; Wildermuth 2010). In each of these biotroph-plant interactions, a localized carbon sink is established and enhanced metabolic rates are likely required to support the nutrient demands of the biotroph (Wildermuth 2010). In addition, when assessed, chromatin in endoreduplicated cells remained largely decondensed, and there was active gene expression. Interestingly, transcriptional analysis of endoreduplicated cells in the G. orontii-Arabidopsis, root nematode-Arabidopsis, and Sinorhizobium sp.-Medicago sp. interactions showed a similar impact on primary host metabolism (Wildermuth 2010). Furthermore, some of these metabolic shifts (e.g., to fermentation) have previously been associated with enhanced metabolic rates in other systems. This suggests that both a net increase in transcription and translation as well as a shift in primary metabolism in endoreduplicated cells may be employed to support the high metabolic demands imposed by these biotrophs.

In plants, cell cycle and differentiation responses are tightly integrated and linked to organogenesis and development (e.g., endosperm, trichomes, and fruit), with endoreduplication often preceding expansion and differentiation (Breuer et al. 2010). Indeed, the same ploidy-associated metabolic processes identified as preferentially upregulated in the G. orontii-Arabidopsis and other biotroph-plant interactions (above) are also upregulated in trichomes and maize endosperm (Wildermuth 2010). Similar to plant cells, endoreduplication is observed in animal cells that provide nutrients to developing embryos (e.g., in fruit fly nurse cells and rodent trophoblast giant cells) and in cells that require high metabolic capacity for rapid growth (e.g., in fruit fly larval cells) (Lee et al. 2009).

We described four PM compatibility factors (MYB3R4, PMR5, PMR6, and PUX2) that influence mesophyll cell ploidy at the PM infection site (Fig. 3). The extent of PM growth and reproduction at $5 \mathrm{dpi}$, assessed as conidiophores per colony, was highly correlated with the mesophyll cell ploidy index, indicating that mesophyll cell ploidy at the PM infection site is a PM-susceptibility determinant. Furthermore, the influence of (induced) host ploidy on PM reproduction can be described using a linear equation (Fig. 3). Therefore, it could potentially be incorporated into mechanistic models of PM disease outcome that integrate host genotype information (e.g., as a parameter affecting spore production) (Calonnec et al. 2008). Though it has not yet been examined, we anticipate that compatible PM fungi will also induce later-stage site-specific mesophyll cell endoreduplication in crops with low basal ploidy levels, such as barley, to increase gene copy number and enhance nutrient supply for the fungus. This would be consistent with observations that the degree of endoploidy in cells of organs with high metabolic capacity is anti-correlated with basal ploidy (Nagl 1976).

\section{PM compatibility factors influencing (induced) cell ploidy likely affect G2/M progression.}

Localized PM-induced mesophyll cell endoreduplication in mature Arabidopsis leaves requires i) cell cycle re-entry, ii) DNA replication and passage through the DNA checkpoint control, and iii) failure to proceed through G2 to mitosis (M) and cell division (Fig. 5). The four identified compatibility factors (MYB3R4, PUX2, PMR5, and PMR6) are members of distinct plant-specific or shared eukaryotic protein families (Fig. 3); however, as discussed below, they all appear to affect progression from G2 to mitosis and cell division.

Plant MYB3R proteins are homologous to animal MYB proteins that function in cell cycle regulation (Berckmans and De Veylder 2009; Ito 2005). Similar to plants, animal MYBs functioning in a multi-protein complex can act as transcriptional activators or repressors (Georlette et al. 2007). Though best known as an activator of mitosis and cytokinesis, MYB3R4 can also promote endoreduplication (Chandran et al. 2010). In the myb3r4 mutant, induced endoreduplication was abolished and the plants supported less PM growth and reproduction (Chandran et al. 2010) (Figs. 2 and 3). Because MYB3R4 was specifically induced at the site of PM infection, with known mitosis-associated targets downregulated (Chandran et al. 2010), it appears to be a major regulator of localized PMinduced mesophyll endoreduplication in WT plants.

PUX2 affected mesophyll ploidy at the PM feeding site through its impact on basal mesophyll cell ploidy. PUX2 is 1 of 15 Arabidopsis proteins containing a ubiquitin-like UBX domain. PUX2 and PUX1 co-localize and interact with the conserved and essential AAA-ATPase CDC48 at the cell division plane (Rancour et al. 2004). The cellular functions of the molecular chaperone CDC48(p97/VCP) are facilitated by its interaction with UBX-containing adapter proteins (Schuberth and Buchberger 2008). In plants, CDC48 is critical for cell division, expansion, and differentiation (Park et al. 2008). Therefore, PUX2 might directly promote endoreduplication associated with cell expansion and differentiation. Alternatively, the pux2 ploidy phenotype could reflect the perturbance of tightly regulated and highly integrated UBX-CDC48 interactions. In contrast to PMR6, which also influences basal mesophyll cell ploidy, mutations in $P U X 2$ do not alter rosette diameter or leaf size (Chandran et al. 2009).

Although the biochemical function of PMR5 is unknown, it is a member of the plant-specific trichome birefringence-like (TBL) family. TBL family members contain an N-terminal transmembrane domain, a TBL domain, and a C-terminal DUF231 domain. Altered cell wall composition has been reported for mutants in a number of TBL, including PMR5 (Bischoff et al. 2010; Gille et al. 2011), with xyloglucan $O$ acetyltransferase function assigned to AXY4 and AXY4-like (Gille et al. 2011). PMR6 contains a pectate lyase-like domain, though pectate lyase activity has not been reported (Vogel et al. 2002). Because of the predicted $\mathrm{N}$-terminal endoplasmic reticulum transport sequence and C-terminal glycosyl-phosphatidylinositol modification site, PMR6 is thought to be attached to the exterior surface of the plasma membrane. Both pmr5 and pmr6 mutants exhibit altered leaf cell wall composition and smaller rosette diameters than the WT, with the defect attributed to reduced cell expansion (Vogel et al. 2002, 2004). We found PMR5 and PMR6 expression to be highly correlated, assessed using ATTED-II, indicating that they act in the same transcriptional network. The intersection of the top 300 correlated genes with PMR5 and PMR6 resulted in a set of 25 genes, 
most enriched in the category Biogenesis of Cellular Compartments (cytoskeleton) with a $P$ value of 4E-04 (Supplementary Table S6). Only one gene in this set of 25, the gibberellinregulated family protein encoded by At5g14920, overlapped with the Interaction with the Environment subset of pmr5 array data. Despite the similar phenotypes and coordinated transcription, pmr5 pmr6 double mutant analysis showed their impact on assessed phenotypes to be synergistic (Vogel et al. 2004). Accordingly, we found both pmr5 and pmr6 to exhibit reduced mesophyll ploidy at the PM feeding site (Fig. 3); however, they differed in whether basal ploidy or the PM-induced increase in ploidy was altered.

Though it is possible that altered cell wall composition directly affects ploidy (for example, by limiting cell expansion or cytokinesis), our microarray analysis of pmr5 suggested a larger role for PMR5 in coordinating differentiation and cell cycle responses via HD-Zip and MYB3R transcription factors (discussed above; Fig. 4C), with an associated impact on cell wall composition. In developing leaves, cell division and endoreduplication (associated with cell expansion) are tightly integrated and exhibit basipetal polarity (Byrne 2005; Gutierrez 2009). The slightly cupped leaf phenotypes of pmr5 and pmr6 (Vogel et al. 2002, 2004) are indicative of miscoordinated differentiation and cell cycle responses, consistent with our expression analyses.

MYB3R transcription factors have complementary and competing functions in regulating the cell cycle which, in some cases, may be dependent upon the phosphorylation status of the protein (Ito 2005; Wildermuth 2010). We proposed that inhibition of aspects of MYB3R-dependent cell cycle responses via PMR5 facilitates expression of the unphosphorylated form of MYB3R4 at the PM-infection site (Fig. 4D) which, in turn, promotes localized endoreduplication and the growth and reproduction of the PM. In pmr5, the inappropriate expression of HD-ZIP and MYB3R transcription factors and the associated regulated genes could limit the amount or function of unphosphorylated MYB3R4 at the infection site, resulting in abrogated PM-induced endoreduplication.

If PMR5 affects PM growth and reproduction solely via its impact on MYB3R4 and infected mesophyll ploidy, we would expect the mean infected ploidy index and extent of PM growth and reproduction of pmr5 to equal that of myb3r4. However, the mean infected ploidy index and PM growth and reproduction of myb3r4 was greater than pmr5 (Fig. 3B). Neither mutant exhibited PM-induced endoreduplication or a statistically significant difference from the WT in basal ploidy index. However, $4 \%$ of mesophyll cell nuclei in uninoculated leaves of myb3r4 were 64C (Fig. 2), whereas the WT and the other mutants had no mesophyll cell nuclei of $64 \mathrm{C}$ prior to inoculation. Therefore, this small number of cells with higher ploidy in myb3r4 could allow for enhanced PM growth and reproduction in the absence of induced endoreduplication. Disruption of MYB3R4 likely affects the integrated function of the five Arabidopsis MYB3R genes through leaf development, resulting in the slight alteration in basal mesophyll cell ploidy distribution for myb3r4 but not pmr5. Restoration of WT MYB3R4 function in $p m r 5$ has been complicated by the dual function of MYB3R4, the highly localized nature of PMinduced MYB3R4 expression, and the competing and complementary functions of the MYB3R proteins.

In addition, our microarray data indicated that $10 \%$ of genes with altered expression in pmr5 were associated with Interaction with the Environment. This raises the possibility that elevated basal defense gene expression (e.g., by SA and ET) may also contribute to PM resistance of pmr5. However, a number of findings argue against this possibility. Most importantly, $\mathrm{PM}$ resistance of pmr5 (and pmr6) was found to be independ- ent of SA, ET, and JA signaling pathways in double mutant analyses (Vogel et al. 2002, 2004). Second, PM growth and reproduction was highly correlated with (induced) mesophyll ploidy index for the set of mutants including pmr5 and pmr6, indicating that altered ploidy was responsible for the PM phenotype (Fig. 3B). Finally, the pmr5 microarray data did not include a strong basal elevated defense signature. Instead, it showed low-level enhanced expression for a small number of genes in diverse hormone and defense categories.

It is interesting that the four identified factors appear to affect progression to $\mathrm{M}$. However, factors initially associated with one phase of the cell cycle often also impact another (Gutierrez 2009). For example, the cyclin-dependent kinase inhibitor KRP2 influences both G1/S and G2/M progressions. Moreover, though we identified PMR5 as acting at the G2/M progression, many of the cell cycle-associated genes with altered expression in pmr5 are also associated with $\mathrm{G} 1 / \mathrm{S}$, including KRP2 and PROLIFERA (Gutierrez 2009). We anticipate that additional compatibility factors will be identified that act both upstream (to mediate cell cycle reentry) and downstream (resulting in enhanced metabolism) of the factors described herein.

By contrast, we do not anticipate host factors associated with DNA damage repair or DNA damage checkpoint control to be PM compatibility factors because genotoxic stress is not associated with successful infection by adapted PM fungi, which suppress defense responses, including cell death. Genotoxic stress, including that associated with a resistant interaction, can result in endoreduplication, presumably to buffer against deleterious mutations or facilitate a reset of chromatin status (Adachi et al. 2011; Cortes et al. 2004; Lee et al. 2009). In addition, endoreduplication may accompany cytoskeletal rearrangement or perturbations induced by some pathogens or chemical treatments. The features of these endoreduplication responses can differ from endoreduplication as part of normal growth and development (Cortes et al. 2004; Lee et al. 2009). Accordingly, a study of early PM resistance responses in barley using a resistant barley cultivar found epidermal cells of barley epidermal leaf peels to exhibit enhanced ploidy, concurrent with rapid penetration resistance-associated responses at $2 \mathrm{hpi}$ (Baluska et al. 1995). Infection with a virulent B. graminis pathotype delayed induction (to $22 \mathrm{hpi}$ ) of both responses. Endoreduplicated cells did not exhibit a parallel increase in nuclear size, and chromatin was condensed, consistent with endoreduplication as part of a resistance response; mesophyll cells and time points later than 1 dpi were not examined (Baluska et al. 1995). By contrast, penetrated cells of a susceptible barley cultivar infected with a compatible adapted $B$. graminis pathotype did not exhibit early penetration resistance responses (e.g., focused microtubule rearrangement) at $24 \mathrm{hpi}$ (Hoefle et al. 2011), and we do not anticipate epidermal cell endoreduplication (with features associated with resistance) in this interaction. As mentioned earlier, we do expect to observe endoreduplication in mesophyll cells underlying the haustorial complex-containing epidermal cell in a compatible B. graminisbarley interaction to facilitate fungal nutrient acquisition during its extensive surface growth and reproduction, as we observed in the $G$. orontii-Arabidopsis interaction.

In conclusion, we determined that host cell cycle modulation is a prominent susceptibility determinant of PM growth and reproduction. Furthermore, it has emerged as an important feature of diverse plant biotrophs that establish a sustained site of nutrient acquisition from the host plant (Wildermuth 2010). In addition, a number of bacterial toxins and effectors that interfere with the mammalian host cell cycle, referred to as cyclomodulins, have now been identified (Oswald et al. 2005). Inhibition of progression through $\mathrm{G} 2 / \mathrm{M}$ is critical to endoreduplication associated with the PM-Arabidopsis, root 
nematode-Arabidopsis, and Sinorhizobium sp.-Medicago sp. interactions (De Almeida Engler et al. 2012; Vinardell et al. 2003; Wildermuth 2010). Similarly, cyclomodulins such as the enteropathogenic Escherichia coli effector cycle inhibiting factor and Shigella type III effector IpaB arrest G2/M progression (Iwai et al. 2007; Taieb et al. 2006). Other cyclomodulins are proposed to promote virulence by interfering with normal cell differentiation (Oswald et al. 2005). In the case of the G. orontii-Arabidopsis interaction, the alteration of differentiation and cell cycle responses in pmr5 abrogates PM-induced endoreduplication and later-stage PM growth and reproduction, perhaps due to altered leaf development or polarity or because the target of a PM effector is no longer expressed. Given the tractability of the G. orontii-Arabidopsis system and plasticity of the plant cell cycle, we anticipate the further identification of plant-specific (e.g., PMR5) and shared eukaryotic host factors (e.g., MYB3R4) that modulate the cell cycle. In addition, we are likely to identify factors associated with ploidy-associated enhanced metabolism, an aspect of cell cycle modulation that has received little attention.

\section{MATERIALS AND METHODS}

\section{Plant lines, growth conditions, and PM inoculation.}

Seed for pmr5-1 and pmr6-3 were provided by S. Somerville (University of California, Berkeley). T-DNA insertion lines myb3r4-3 (SALK_116974c) and pux2-1 (SALK_148507) have been described previously (Chandran et al. 2009, 2010). A. thaliana WT Col-O and mutants in the Col-O background were grown in Percival AR66L growth chambers at $22^{\circ} \mathrm{C}, 70 \%$ relative humidity, and a 12-h photoperiod with photosynthetically active radiation $=150 \mu \mathrm{mol} \mathrm{m} \mathrm{m}^{-2} \mathrm{~s}^{-1}$. Plants were inoculated with $G$. orontii MGH isolate, as described (Chandran et al. 2010). Three fully expanded mature leaves were harvested from inoculated and uninoculated plants at the indicated time points for analyses. To visualize and quantify fungal structures, inoculated leaves were stained with trypan blue and mounted in glycerol, as described (Chandran et al. 2009, 2010). For fungal hyphal area measurements, the area of a rectangle incorporating the longest hyphal length was measured from 15 isolated fungal colonies at each time point. Fungal colony shape was fairly consistent at the different time points postinoculation and best represented by a rectangle. All experiments were repeated at least twice, with similar results.

\section{Nuclear DNA quantitation at $G$. orontii infection sites.}

Nuclear DNA quantitation was performed on DAPI-stained leaves with images captured using a Zeiss 510 UV/Vis meta laser-scanning confocal microscope (Carl Zeiss, Inc., Oberkochen, Germany) and analyzed using Imaris (v7.2, Bitplane, Inc., MN) 3D image analysis software, as described (Chandran et al. 2010). DAPI fluorescence values of epidermal and mesophyll cell nuclei were normalized to that of nuclei of reference diploid guard cells from the same image after subtraction of background from each measurement. The boundaries of DAPIstained nuclei were used to measure nuclear volume in Imaris. Two independent experiments gave similar results, with $\geq 30$ nuclei quantified for each time point or genotype.

\section{The pmr5 microarray analysis.}

Raw Arabidopsis Affymetrix ATH1 microarray data for fully expanded leaves of 3-week-old pmr5 and WT Col-O plants is described in National Center for Biotechnology Information Gene Expression Omnibus GSE39708. Expression values $\left(\log _{2}\right)$ for three independent biological replicates per genotype were extracted using robust multiarray analysis using $R$ (v2.14.2). Genes (163 total) with statistically significant differ- ential expression of $\geq 1.5$-fold change between mutant and the WT were selected using an unpaired $t$ test $(P<0.05)$ with the open source software MeV (MultiExperiment Viewer, v 4.8.1) (Saeed et al. 2003) and Benjamini-Hochberg (Benjamini and Hochberg 1995) false-discovery-rate cutoff of 0.05. TAIR10 gene functional descriptions were employed.

\section{ACKNOWLEDGMENTS}

We thank S. Somerville (University of California, Berkeley) for pmr5-1 and pmr6-3 seed and M. Steinwand and S. Hotton and S. Somerville for comments on the manuscript. D. Chandran and J. Rickert were supported by the National Science Foundation (NSF) grant IOS-0958100 to M. C. Wildermuth. C. Cherk was supported, in part, by NSF grant IOS-0929226 to S. Somerville. B. R. Dotson was supported, in part, by an NSF Graduate Fellowship and a Chancellor's Fellowship. D. Chandran and J. Rickert performed all research, except sample preparation for pmr5 microarray and qPCR validation of array (performed by C. Cherk and B. R. Dotson). D. Chandran and M. C. Wildermuth designed the research, analyzed data, and wrote the article.

\section{LITERATURE CITED}

Adachi, S., Minamisawa, K., Okushima, Y., Inagaki, S., Yoshiyama, K., Kondou, Y., Kaminuma, E., Kawashima, M., Toyoda, T., Matsui, M., Kurihara, D., Matsunaga, S., and Umeda, M. 2011. Programmed induction of endoreduplication by DNA double strand breaks in Arabidopsis. Proc. Natl. Acad. Sci. U.S.A. 108:10004-10009.

Baluska, F., Bacigalova, K., Oud, J. L., Hauskrecht, M., and Kubica, S. 1995. Rapid reorganization and microtubular cytoskeleton accompanies early changes in nuclear ploidy and chromatin structure in postmitotic cells of barley leaves infected with powdery mildew. Protoplasma 185:140-151.

Benjamini, Y., and Hochberg, Y. 1995. Controlling the false discovery rate: A practical and powerful approach to multiple testing. J. R. Stat. Soc. B57:289-300.

Berckmans, B., and De Veylder, L. 2009. Transcriptional control of the cell cycle. Curr. Opin. Plant Biol. 12:599-605.

Bischoff, V., Selbig, J., and Scheible, W. R. 2010. Involvement of TBL/DUF231 proteins in cell wall biology. Plant Signal. Behav. 5:1057-1059

Breuer, C., Ishida, T., and Sugimoto, K. 2010. Developmental control of endocycles and cell growth in plants. Curr. Opin. Plant Biol. 13:654660.

Byrne, M. E. 2005. Networks in leaf development. Curr. Opin. Plant Biol. 8:59-66.

Calonnec, A., Cartolaro, P., Naulin, J.-M., Bailey, D., and Langlais, M. 2008. A host-pathogen simulation model: Powdery mildew of grapevine. Plant Pathol. 57:493-508.

Chandran, D., Tai, Y. C., Hather, G., Dewdney, J., Denoux, C., Burgess, D. G., Ausubel, F. M., Speed, T. P., and Wildermuth, M. C. 2009. Temporal global expression data reveals known and novel salicylate-impacted processes and regulators mediating powdery mildew growth and reproduction on Arabidopsis. Plant Physiol. 149:1435-1451.

Chandran, D., Inada, N., Hather, G., Kleindt, C. K., and Wildermuth, M. C. 2010. Laser microdissection of Arabidopsis cells at the powdery mildew infection site reveals site-specific processes and regulators. Proc. Natl. Acad. Sci. U.S.A. 107:460-465.

Consonni, C., Humphry, M. E., Hartmann, H. A., Livaja, M., Durner, J., Westphal, L., Vogel, J., Lipka, V., Kemmerling, B., Schulze-Lefert, P., Somerville, S. C., and Panstruga, R. 2006. Conserved requirement for a plant host cell protein in powdery mildew pathogenesis. Nat. Genet. 38:716-720.

Cortes, F., Mateos, S., Pastor, N., and Dominguez, I. 2004. Toward a comprehensive model for induced endoreduplication. Life Sci. 76:121-135.

De Almeida Engler, J., Kyndt, T., Vieira, P., Van Cappelle, E., Boudolf, V., Sanchez, V., Escobar, C., De Veylder, L., Engler, G., Abad, P., and Gheysen, G. 2012. CCS52 and DEL1 genes are key components of the endocycle in nematode-induced feeding sites. Plant J. 72:185-198.

Dik, A. J., Belanger, R. R., Carver, T. L. W., and Bushnell, W. R. 2002. The Powdery Mildews. American Phytopathological Society, St. Paul, MN, U.S.A.

Eichmann, R., and Huckelhoven, R. 2008. Accommodation of PM fungi in intact plant cells. J. Plant Physiol. 165:5-18.

Ellis, C., and Turner, J. G. 2001. The Arabidopsis mutant cev1 has constitutively active jasmonate and ethylene signal pathways and enhanced resistance to pathogens. Plant Cell 13:1025-1033. 
Georlette, D., Ahn, S., MacAlpine, D. M., Cheung, E., Lewis, P. W., Beall, E. L., Bell, S. P., Speed, T., Manak, J. R., and Botchan, M. R. 2007. Genomic profiling and expression studies reveal both positive and negative activities for the Drosophila Myb MuvB/dREAM complex in proliferating cells. Genes Dev. 21:2880-2896.

Gille, S., de Souza, A., Xiong, G., Benz, M., Cheng, K., Schultink, A., Reca, I.-B., and Pauly, M. 2011. O-acetylation of Arabidopsis hemicellulose xyloglucan requires AXY4 or AXY4L, proteins with a TBL and DUF231 domain. Plant Cell 23:4041-4053.

Gu, Y. Q., Wildermuth, M. C., Chakravarthy, S., Loh, Y. T., Yang, C., He, X., Han, Y., and Martin, G. B. 2002. Tomato transcription factors Pti4 Pti5, and Pti6 activate defense responses in Arabidopsis. Plant Cell $14: 817-831$

Gutierrez, C. 2009. The Arabidopsis cell division cycle. The Arabidopsis Book 7:e0120.

Haga, N., Kato, K., Murase, M., Araki, S., Kubo, M., Demura, T., Suzuki, K., Müller, I., Voss, U., Jürgens, G., and Ito, M. 2007. R1R2R3-Myb proteins positively regulate cytokinesis through activation of KNOLLE transcription in Arabidopsis thaliana. Development 134:1101-1110.

Hoefle, C. Huesmann, C., Schultheiss, H., Bornke, F. Hensel, G., Kumlehn, J., and Huckelhoven, R. 2011. A barley ROP GTPase activating protein associates with microtubules and regulates entry of the barley powdery mildew fungus into leaf epidermal cells. Plant Cell 23:2422-2439.

Ito, M. 2005. Conservation and diversification of three-repeat Myb transcription factors in plants. J. Plant Res. 118:61-69.

Iwai, H., Kim, M., Yoshikawa, Y., Ashida, H., Ogawa, M., Fujita, Y., Muller, D., Kirikae, T., Jackson, P. K., Kotani, S., and Sasakawa, C. 2007. A bacterial effector targets Mad2L2, an APC inhibitor, to modulate host cell cycling. Cell 130:611-623.

Katari, M. S., Nowicki, S. D., Aceituno, F. F., Nero, D., Kelfer, J., Thompson, L. P., Cabello, J. M., Davidson, R. S., Goldberg, A. P., Shasha, D. E., Coruzzi, G. M., and Gutiérrez, R. A. 2010. VirtualPlant: A software platform to support Systems Biology research. Plant Physiol. 152:500-515.

Koh, S., André, A., Edwards, H., Ehrhardt, D., and Somerville, S. 2005. Arabidopsis thaliana subcellular responses to compatible Erysiphe cichoracearum infections. Plant J. 44:516-529.

Lee, H. O., Davidson, J. M., and Duronio, R. J. 2009. Endoreplication: Polyploidy with purpose. Genes Dev. 23:2461-2477.

Melaragno, J. E., Mehrotra, B., and Coleman, A. W. 1993. Relationship between endopolyploidy and cell size in epidermal tissue of Arabidopsis. Plant Cell 5:1661-1668.

Micali, C., Gollner, K., Humphry, M., Consonni, C., and Panstruga, R. 2008. Pages 1-19 in: The Arabidopsis Book. American Society of Plant Biologists, Rockville, MD, U.S.A.

Nagl, W. 1976. DNA endoreduplication and polyteny understood as evolutionary strategies. Nature 261:614-615.

Nishimura, M. T., Stein, M., Hou, B. H., Vogel, J. P., Edwards, H., and Somerville, S. C. 2003. Loss of callose synthase results in salicylic acid-dependent disease resistance. Science 301:969-972.

Obayashi, T., Hayashi, S., Saeki, M., Ohta, H., and Kinoshita, K. 2009. ATTED-II provides coexpressed gene networks for Arabidopsis. Nucleic Acids Res. 37:D987-D991.

Oswald, E., Nougayrede, J. P., Taieb, F., and Sugai, M. 2005. Bacterial toxins that modulate host cell-cycle progression. Curr. Opin. Microbiol. 8:83-91

Park, S., Rancour, D. M., and Bednarek, S. Y. 2008. In planta analysis of the cell cycle-dependent localization of AtCDC48A and its critical roles in cell division, expansion, and differentiation. Plant Physiol. 148:246258

Rancour, D. M., Park, S., Knight, S. D., and Bednarek, S. Y. 2004. Plant UBX-domain containing protein PUX1 regulates the oligomeric structure and activity of Arabidopsis CDC48. J. Biol. Chem. 279:5426454272 .
Reuber, T. L., Plotnikova, J. M., Dewdney, J., Rogers, E. E., Wood, W., and Ausubel, F. M. 1998. Correlation of defense gene induction defects with powdery mildew susceptibility in Arabidopsis enhanced disease susceptibility mutants. Plant J. 16:473-485.

Saeed, A. I., Sharov, V., White, J., Li, J., Liang, W., Bhagabati, N., Braisted, J., Klapa, M., Currier, T., Thiagarajan, M., Sturn, A., Snuffin, M., Rezantsev, A., Popov, D., Ryltsov, A., Kostukovich, E., Borisovsky, I., Liu, Z., Vinsavich, A., Trush, V., and Quackenbush, J. 2003. TM4: A free, open-source system for microarray data management and analysis. Biotechniques 34:374-378.

Schuberth, C., and Buchberger, A. 2008. UBX domain proteins; major regulators of the AAA ATPase Cdc48/p97. Cell Mol. Life Sci. 65:23602371

Spanu, P. D., Abbott, J. C., Amselem, J., Burgis, T. A., Soanes, D. M., Stüber, K., Ver Loren van Themaat, E., Brown, J. K., Butcher, S. A., Gurr, S. J., Lebrun, M. H., Ridout, C. J., Schulze-Lefert, P., Talbot, N. J., Ahmadinejad, N., Ametz, C., Barton, G. R., Benjdia, M., Bidzinski, P., Bindschedler, L. V., Both, M., Brewer, M. T., Cadle-Davidson, L., Cadle-Davidson, M. M., Collemare, J., Cramer, R., Frenkel, O., Godfrey, D., Harriman, J., Hoede, C., King, B. C., Klages, S., Kleemann, J., Knoll, D., Koti, P. S., Kreplak, J., López-Ruiz, F. J., Lu, X. ,Maekawa, T., Mahanil, S., Micali, C., Milgroom, M. G., Montana, G., Noir, S., O'Connell, R. J., Oberhaensli, S., Parlange, F., Pedersen, C., Quesneville, H., Reinhardt, R., Rott, M., Sacristán, S., Schmidt, S. M., Schön, M., Skamnioti, P., Sommer, H., Stephens, A., Takahara, H., Thordal-Christensen, H., Vigouroux, M., Wessling, R., Wicker, T., and Panstruga, R. 2010. Genome expansion and gene loss in powdery mildew fungi reveal tradeoffs in extreme parasitism. Science 330:15431546

Taieb, F., Nougayrede, J. P., Watrin, C., Samba-Louaka, A., and Oswald, E. 2006. Escherichia coli cyclomodulin cif induces G2 arrest of the host cell cycle without activation of the DNA-damage checkpoint signaling pathway. Cell. Microbiol. 8:1910-1921.

Tessadori, F., Chupeau, M. C., Chupeau, Y., Knip, M., Germann, S., van Driel, R., Fransz, P., and Gaudin, V. 2007. Large-scale dissociation and sequential reassembly of pericentric heterochromatin in dedifferentiated Arabidopsis cells. J. Cell Sci. 120:1200-1208.

Vinardell, J. M., Fedorova, E., Cebolla, A., Kevei, Z., Horvath, G. Kelemen, Z., Tarayre, S., Roudier, F., Mergaert, P., Kondorosi, A., and Kondorosi, E. 2003. Endoreduplication mediated by the anaphasepromoting complex activator CCS52A is required for symbiotic cell differentiation in Medicago truncatula nodules. Plant Cell 15:20932105

Vogel, J. P., Raab, T. K., Schiff, C., and Somerville, S. C. 2002. PMR6, a pectate lyase-like gene required for powdery mildew susceptibility in Arabidopsis. Plant Cell 14:2095-2106.

Vogel, J. P., Raab, T. K., Somerville, C. R., and Somerville, S. C. 2004 Mutations in PMR5 result in powdery mildew resistance and altered cell wall composition. Plant J. 40:968-978.

Wildermuth, M. C. 2010. Modulation of host nuclear ploidy: A common plant biotroph mechanism. Curr. Opin. Plant Biol. 13:449-458.

Wildermuth, M. C., Dewdney, J., Wu, G., and Ausubel, F. M. 2001. Isochorismate synthase is required to synthesize salicylic acid for plant defense. Nature 414:562-565.

Zambelli, F., Pesole, G., and Pavesi, G. 2009. Pscan: Finding over-represented transcription factor binding site motifs in sequences from co-regulated or co-expressed genes. Nucleic Acids Res. 37:W247W252.

\section{AUTHOR-RECOMMENDED INTERNET RESOURCES}

Virtual Plant database: www.virtualplant.org

The Arabidopsis Information Resource (TAIR) database: www.arabidopsis.org 